\title{
Image-based quantification of Fusarium wilt severity in banana
}

\author{
Ryan Orr ${ }^{1}$ (D) Anthony Pattison ${ }^{2} \cdot$ David East $^{2} \cdot$ Noeleen Warman $^{3} \cdot$ Wayne O'Neill $^{3} \cdot$ Elizabeth Czislowski $^{4}$. \\ Paul N. Nelson ${ }^{1}$
}

Received: 4 December 2018 / Accepted: 1 May 2019/Published online: 23 May 2019

(C) Australasian Plant Pathology Society Inc. 2019

\begin{abstract}
The severity of Fusarium wilt of bananas has long been classified based on visual assessment of necrosis in rhizome or pseudostem cross-sections. The improved method proposed here uses digital image analysis to quantify the proportion of rhizome tissue that is necrotic. It agrees well with visual classification, but provides greater reproducibility, precision and statistical power.
\end{abstract}

Keywords Fusarium oxysporum f. sp. cubense $\cdot$ Panama disease $\cdot$ Disease severity $\cdot$ Image analysis $\cdot$ Method

Fusarium wilt of banana, or Panama disease, is rapidly spreading throughout the world. As the impact of this disease increases, so too does the need to quantify disease severity in research projects assessing prevention and treatment options. The method proposed here has been designed for Fusarium wilt of banana, but it could also be used to assess Fusarium wilt of other crops, or other plant diseases resulting in tissue discoloration.

Existing methods for quantifying Fusarium wilt severity visually categorize cross sections based on the level of vascular tissue discoloration of rhizome and sometimes pseudostem or root tissue (Carlier et al. 2003; Orjeda 1998; Peng et al. 1999; Smith et al. 2008; Viljoen et al. 2016; Zadoks and Schein 1979; Zuo et al. 2018). Fusarium oxysporum f. sp. cubense (Foc), the causal organism of Fusarium wilt, initially infects the roots of bananas, moves through the rhizome, causing rhizome necrosis and discoloration, before moving into

Ryan Orr

ryan.orr@jcu.edu.au

1 College of Science and Engineering, James Cook University, Cairns, Qld 4878, Australia

2 QLD Department of Agriculture and Fisheries, South Johnstone, Qld 4859, Australia

3 QLD Department of Agriculture and Fisheries, Dutton Park, Qld 4102, Australia

4 School of Agriculture and Food Sciences, The University of Queensland, St. Lucia, Qld 4072, Australia the pseudostem and leaves (Warman and Aitken 2018). Therefore, rhizome tissue colour or necrosis is a suitable proxy for disease severity (Ploetz 2015), however the causative organism should still be confirmed using traditional methods (Puhalla 1985). The method presented here has two advantages over current methods: 1) precision and thus statistical power are increased as the variable measured is continuous rather than discrete 2) subjectivity and human error are reduced by using computerised image analysis rather than the human eye to determine the proportion of tissue that is discolored.

Existing methods typically use a 6-class scale with the first class for no disease presence. Each rhizome cross section is scored and then scores are averaged across all sections from an individual plant. The classification system from Viljoen et al. (2016) is: $1=$ No symptoms; $2=$ Few internal spots; $3=<1 / 3$ Discoloured tissue; $4=1 /$ 3-2/3 Discoloured tissue; $5=>2 / 3$ Discoloured tissue; $6=$ Entire inner rhizome discoloured. Thus, samples with slightly different discoloration can have severity scores differing by $33 \%$ and samples with the same score may differ by up to $32 \%$. By using a continuous scale that has high reproducibility the precision can be improved.

The method proposed here quantifies discoloration of the central region of vascular tissue in the rhizome as follows. Three transverse sections of the rhizome are taken, one quarter, half, and three quarters up, after the removal of all soil and roots. A photograph is taken of the sections, including a gray scale reference, and 
converted to a binary image based on the differentiation of healthy and diseased tissue (white and black pixels respectively). Conversion to binary values removes any variations in colour unrelated to disease. The gray scale reference allows for comparison between photos taken under different light conditions. The pixel values for the area of interest in each rhizome section are averaged. These section values are then averaged to determine an average pixel value for all sections of rhizome which is divided by the pixel value for diseased tissue to determine the proportion of necrotic tissue for each plant rhizome. This analysis is possible using a variety of image analysis programs, but we provide a step-bystep guide for the open source image analysis program ImageJ (see Appendix).

We compared the digital image analysis method with an existing visual rating method from Viljoen et al. (2016) in four banana pot experiments using either Ducasse (Musa ABB, synonym Pisang Awak) inoculated with Foc Race 1 (Vegetative Compatibility Group (VCG) 0124, BRIP 43996 and 61,873) or Williams (Musa AAA, Cavendish subgroup) inoculated with Foc Subtropical Race 4 (VCG 0120, BRIP 40272 and 42,107), so that internal disease symptoms spanned the entire range of disease severity. Samples of discoloured tissue were previously confirmed positive with initial Foc inoculum, either VCG 0124 or VCG 0120 , using purified isolates of the fungus re-isolated from banana rhizomes (Puhalla 1985). Furthermore, a 660 base pair region of the translation elongation factor $(\mathrm{EF} 1-\alpha)$ from each isolate was amplified, sequenced and compared with Foc sequences from previous studies with $100 \%$ sequence identity (Czislowski et al. 2018; Fourie et al. 2009; O’Donnell et al. 1998). The sequences generated in this study are available on GenBank (accessions MK767022-MK767025). Finally, tissue discolouration was absent in plants unless they received Foc inoculum. There was close correlation between the methods, and the absence of disease was well classified in both methods (Fig. 1). The quadratic best-fit (Fig. 1) indicated that compared with the proposed method, severity scores between 4.3 and 6 were classified too high, and values between 2.3 and 1.3 were classified too low using the Viljoen et al. (2016) method (Fig. 1). This may result from researcher's inadvertently increasing variability in the classification of their samples despite the presence of a limited range of disease severity. It can also be observed that at high disease severity there was greater differentiation amongst samples using the imaging method compared to use of disease severity classes, indicating the imaging method was more effective at differentiation between samples with high levels of tissue discolouration.

By increasing the precision of disease severity rating, the new method increases statistical power, allowing greater confidence in experimental results, or reducing the number of samples
Fig. 1 A comparison of disease severity as determined using the 6-class scale of Viljoen et al. (2016) and the method proposed here (proportion of area), across four pot experiments $(\mathrm{y}=$ $-0.0004 \times^{2}+0.0876 \mathrm{x}+1.1358$ $\left.R^{2}=0.9641\right)$. Rectangles show the range of values using the new method for five ranges of the class method and the shaded region shows the theoretical zone of correspondence between the two methods

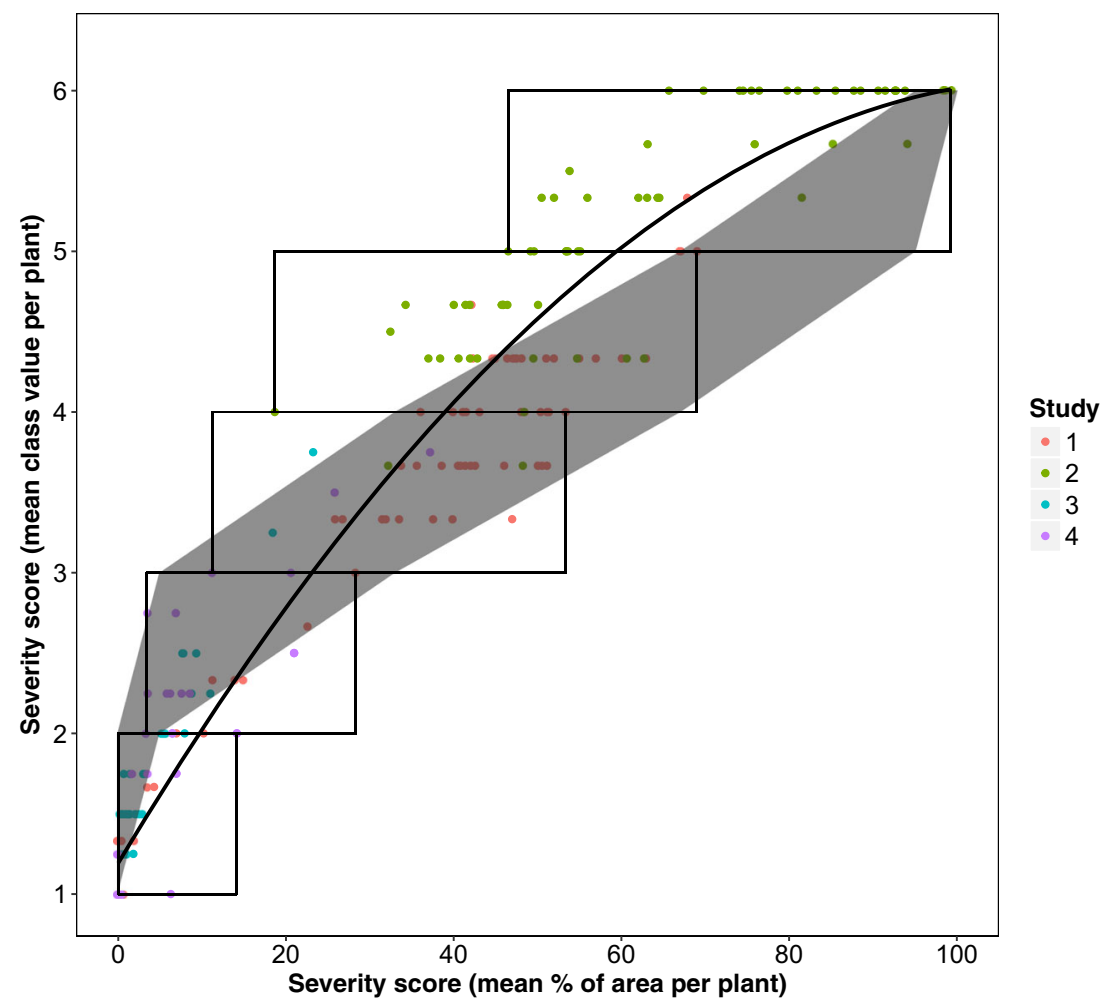


required for a given level of confidence. Furthermore, by decreasing the requirement for human decision making and classification it is likely that better comparison between studies can be made.

Acknowledgements This project has been funded by Hort Innovation, using the Hort Innovation banana research and development levy, coinvestment from Queensland Government and contributions from the Australian Government. Hort Innovation is the grower-owned, not-forprofit research and development corporation for Australian horticulture.

\section{Appendix}

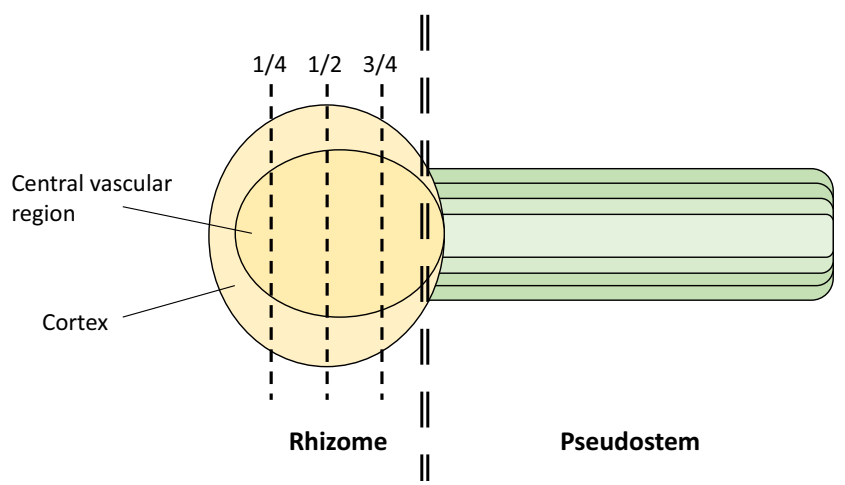

Fig. 2 Following removal of roots, the rhizome should be separated from the pseudostem and transverse sections of the rhizome taken at $1 / 4,1 / 2$ and $3 / 4$ of the distance from the bottom of the rhizome
Notes for photographing sections:

- Good focus is required for maximum effectiveness

- Avoid image distortion by taking photos from directly overhead

- Consistent light level simplifies analysis

- Avoid partial shadows or light variation within the image

- Inclusion of an 8-bit grayscale calibration allows for consistent comparison between photos

- Rhizome sections must be free from dark material such as soil, leaf litter.

All image processing can be performed using ImageJ $\mathrm{v} 1.51 \mathrm{t}$, as follows.

1. Open photo. File $>$ Open.

2. Create duplicate. Image $>$ Duplicate. Keep both visible on your screen, this makes for easy comparison between the processed and unprocessed image.

3. Convert image duplicate to 8-bit black and white. Image $>$ Type $>8$-bit.

4. Convert duplicate to binary. Image $>$ Adjust $>$ Threshold $>$ Apply. Set threshold value so that areas of disease are red and areas of non-disease are not red.

Note: Threshold value may change between images due to variance in lighting, use the in-photo grayscale to establish value, typically differentiation between 108 and 128 pixel value on the calibration strip.

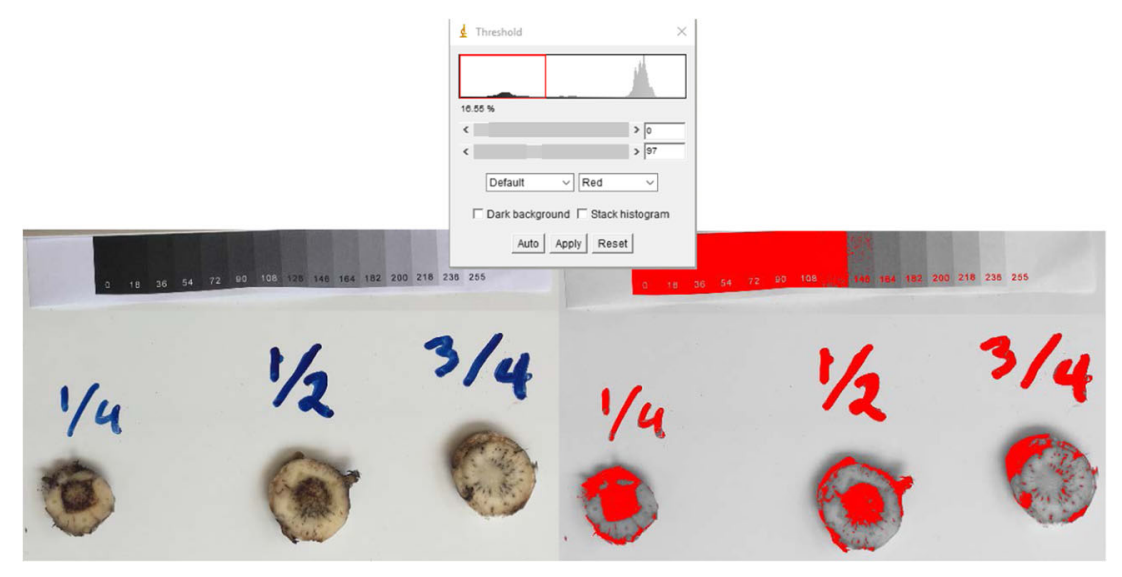

5. Setup for measurement. Analyze $>$ Set Measurements $>$ Ensure "Mean gray value", "Display label" and "Add to overlay" are checked. Set Redirect to: Binary image.

6. Measure values: On the non-binary image use the "Freehand selections" tool (fourth from left on toolbar) to outline central vascular region of rhizome in one polygon. The oval or elliptical selector is not suitable as the relevant tissue is rarely a perfect oval.

7. Measure value for one section: Analyze $>$ Measure $(C t r l+M)$. Record the mean pixel value for the selected area for further calculation. 


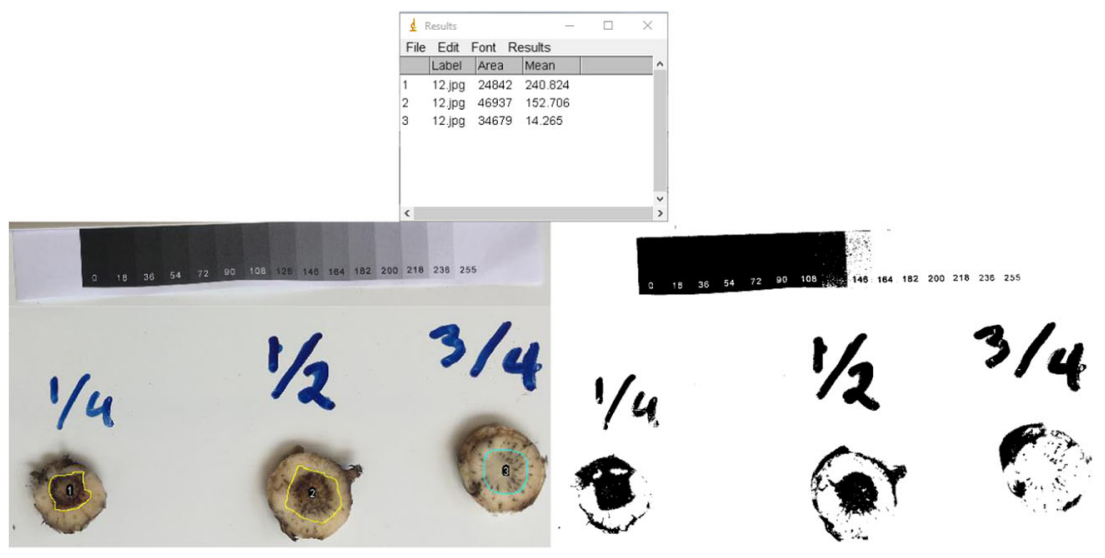

8. Repeat outlining and measurement steps for other sections.

9. Data can then be transposed for analysis. As the mean value is measured on a binary image with white $(0)$ and black (255) values, the mean for the selection is the ratio of discoloured to non-discoloured tissue. The rhizome section values can be averaged and then divided by 255 (value if all pixels are black) to obtain the proportion of necrosis for each plant.

\section{References}

Carlier J, De Waele D, Escalant J (2003) Global evaluation of Musa germplasm for resistance to Fusarium wilt, Mycosphaerella leaf spot diseases and nematodes. Performance evaluation. INIBAP Technical Guidelines 7

Czislowski E, Fraser-Smith S, Zander M, O'Neill WT, Meldrum RA, Tran-Nguyen LTT, Batley J, Aitken EAB (2018) Investigation of the diversity of effector genes in the banana pathogen, Fusarium oxysporum f. sp. cubense, reveals evidence of horizontal gene transfer. Mol Plant Pathol 19:1155-1171. https://doi.org/10.1111/mpp. 12594

Fourie G, Steenkamp ET, Gordon TR, Viljoen A (2009) Evolutionary relationships among the Fusarium oxysporum $\mathrm{f}$. sp. cubense vegetative compatibility groups. Appl Environ Microbiol 75:4770-4781. https://doi.org/10.1128/AEM.00370-09

O’Donnell K, Kistler HC, Cigelnik E, Ploetz RC (1998) Multiple evolutionary origins of the fungus causing Panama disease of banana: concordant evidence from nuclear and mitochondrial gene genealogies. Proc Natl Acad Sci 95:2044-2049. https://oi.org/10.1073/ pnas.95.5.2044

Orjeda G (1998) Evaluation of Musa germplasm for resistance to Sigatoka diseases and Fusarium wilt. vol 634.77294/O69. IPGRI/ INIBAP,

Peng HX, Sivasithamparam K, Turner DW (1999) Chlamydospore germination and Fusarium wilt of banana plantlets in suppressive and conducive soils are affected by physical and chemical factors. Soil Biol Biochem 31:1363-1374

Ploetz RC (2015) Fusarium wilt of Banana. Phytopathology 105:15121521. https://doi.org/10.1094/PHYTO-04-15-0101-RVW

Puhalla JE (1985) Classification of strains of Fusarium oxysporum on the basis of vegetative compatibility. Can J Bot 63:179-183. https://doi. org/10.1139/b85-020

Smith LJ, Smith MK, Tree D, O'Keefe D, Galea VJ (2008) Development of a small-plant bioassay to assess banana grown from tissue culture for consistent infection by Fusarium oxysporum f. sp. cubense. Australas Plant Pathol 37:171-179. https://doi.org/10.1071/ ap08006

Viljoen A et al. (2016) Banana pests and diseases: field guide for disease diagnostics and data collection

Warman NM, Aitken EAB (2018) The movement of Fusarium oxysporum f.sp. cubense (sub-tropical race 4) in susceptible cultivars of Banana. Front Plant Sci 9. https://doi.org/10.3389/fpls.2018. 01748

Zadoks JC, Schein RD (1979) Epidemiology and plant disease management. Oxford University Press Inc, New York

Zuo C, Deng G, Li B, Huo H, Li C, Hu C, Kuang R, Yang Q, Dong T, Sheng O, Yi G (2018) Germplasm screening of Musa spp. for resistance to Fusarium oxysporum f. sp. cubense tropical race 4 (Foc TR4). Eur J Plant Pathol 151:723-734. https://doi.org/10.1007/ s10658-017-1406-3 